MITSUBISHI ELECTRIC RESEARCH LABORATORIES

http://www.merl.com

\title{
A Rapid Acquisition Technique for Impulse Radio
}

Gezici, S.; Fishler, E.; Kobayashi, H.; Poor, H.V.

TR2003-46 August 2003

\begin{abstract}
A novel rapid acquisition algorithm, called Sequential Block Search, is proposed for impulse radio systems, which can reduce the mean acquisition time considerably. The algorithm is compared to the conventional serial search algorithm and output statistics for both algorithms are derived for a binary phase-shift keyed random time hopping impulse radio system. The mean acquisition time formulas are obtained using the signal flow graph approach. The simulations indicate the superiority of the rapid acquisition algorithm over the serial search technique with a modest increase in the complexity.
\end{abstract}

This work may not be copied or reproduced in whole or in part for any commercial purpose. Permission to copy in whole or in part without payment of fee is granted for nonprofit educational and research purposes provided that all such whole or partial copies include the following: a notice that such copying is by permission of Mitsubishi Electric Research Laboratories, Inc.; an acknowledgment of the authors and individual contributions to the work; and all applicable portions of the copyright notice. Copying, reproduction, or republishing for any other purpose shall require a license with payment of fee to Mitsubishi Electric Research Laboratories, Inc. All rights reserved.

Copyright (C) Mitsubishi Electric Research Laboratories, Inc., 2003

201 Broadway, Cambridge, Massachusetts 02139 



\section{Publication History:}

1. First printing, TR-2003-46, August 2003 


\section{A Rapid Acquisition Technique for Impulse Radio}

\author{
Sinan Gezici, Eran Fishler, Hisashi Kobayashi and H. Vincent Poor ${ }^{1}$ \\ Department of Electrical Engineering \\ School of Engineering and Applied Science \\ Princeton University, Princeton, NJ 08544 \\ \{sgezici,efishler,hisashi,poor\}@ princeton.edu
}

\author{
Andreas F. Molisch ${ }^{2}$ \\ Mitsubishi Electric Research Laboratories \\ 201 Broadway \\ Cambridge, MA 02139 \\ Andreas.Molisch@ieee.org
}

\begin{abstract}
A novel rapid acquisition algorithm, called Sequential Block Search, is proposed for impulse radio systems, which can reduce the mean acquisition time considerably. The algorithm is compared to the conventional serial search algorithm and output statistics for both algorithms are derived for a binary phase-shift keyed random time hopping impulse radio system. The mean acquisition time formulas are obtained using the signal flow graph approach. The simulations indicate the superiority of the rapid acquisition algorithm over the serial search technique with a modest increase in the complexity.
\end{abstract}

\section{INTRODUCTION}

In impulse radio communication systems, the acquisition of an incoming signal is critical in de-modulation of the signal which often takes the form of PPM (pulse position modulation) or BPSK (binary phase shift keying). Because of the high resolution in time required to locate narrow pulses employed in ultra-wideband (UWB) systems, a considerable number of possible pulse positions must be searched in order to acquire the received signal. Therefore, a rapid acquisition algorithm is very important in UWB communications.

The role of an acquisition technique is essentially to determine the relative delay of the received signal with respect to a template signal in the receiver. The conventional technique to achieve this is what is known as the serial search algorithm [1], in which the received signal is correlated with the template signal and the correlation output is compared to a threshold. If the output is lower than the threshold, the template signal is shifted by an amount comparable to pulse interval and is correlated with the received signal. This procedure continues until an output exceeds the threshold. This serial search algorithm may take a long time, thus, some quick algorithm is called for.

We propose a rapid acquisition algorithm, called Sequential Block Search (SBS), which can considerably reduce the mean acquisition time, while maintaining the receiver complexity comparable to the serial search algorithm.

The SBS algorithm first aims to determine a subregion where the signal delay is likely to exist. Then, this subregion is examined in a finer detail to find the exact position of the signal. In order to perform the first step quickly, we define a new template signal. The correlation of the received signal with this template signal effectively adds a number of serial search

\footnotetext{
${ }^{1}$ This research is supported in part by the National Science Foundation under grant CCR-99-79361, and in part by the New Jersey Center for Wireless Telecommunications.

${ }^{2}$ Also with the Department of Electroscience, Lund University.
}

correlation outputs within a certain block in time. This output from the block is then used as a criterion to decide if the block contains the signal or not. Whenever the block output exceeds a specified threshold, we apply the conventional serial search algorithm within this subregion corresponding to the block.

The remainder of the paper is organized as follows. Section II defines the signal structure used during the acquisition process. SBS algorithm is explained in Section III. Then, statistics of correlation outputs are derived in Section IV, which is followed by acquisition time analysis and simulation results in Section V. Finally, some concluding remarks are made in Section VI.

\section{Signal Model}

Consider a binary phase-shift keyed random time hopping impulse radio (TH-IR) system where the transmitted signal from user $k$ in an $N_{u}$-user setting is represented by the following model [3]:

$$
s_{t r}^{k}(t)=\sum_{j=-\infty}^{\infty} d_{j}^{k} b_{\left\lfloor j / N_{f}\right\rfloor}^{k} w_{t x}\left(t-j T_{f}-c_{j}^{k} T_{c}\right),
$$

where $w_{t x}$ is the transmitted unit-energy pulse, $T_{f}$ is the average pulse repetition time, $N_{f}$ is the number of pulses representing one information symbol and $b_{\left\lfloor j / N_{f}\right\rfloor}^{k} \in\{+1,-1\}$ is the information symbol transmitted by user $k$. In order to allow the channel to be exploited by many users and avoid catastrophic collisions, a pseudo-random sequence $\left\{c_{j}^{k}\right\}$, where $c_{j}^{k} \in\left\{0,1, \ldots, N_{c}-1\right\}$, is assigned to each user. This sequence is called time hopping sequence and provides an additional time shift of $c_{j}^{k} T_{c}$ seconds to the $j$ th pulse of the $k$ th user where $T_{c}$ is the chip interval and is chosen to satisfy $T_{c} \leq T_{f} / N_{c}$ in order to prevent the pulses from overlapping.

In this paper, we consider coded IR systems where $d_{j}^{k}$,s are binary random variables, independent for $(j, k) \neq(i, l)$, taking each of the values \pm 1 with probability $1 / 2$ [3]. This system can be regarded as a random Direct Sequence CDMA (RCDMA) system with $T_{f}=T_{c}$ and $N_{f}$ representing the processing gain.

Define a sequence $\left\{s_{j}^{k}\right\}$ as follows

$$
s_{j}^{k}=\left\{\begin{array}{cc}
d_{\left\lfloor j / N_{c}\right\rfloor}^{k}, & j-N_{f}\left\lfloor j / N_{c}\right\rfloor=c_{\left\lfloor j / N_{c}\right\rfloor}^{k} \\
0, & \text { otherwise. }
\end{array}\right.
$$

Then, assuming $T_{f} / T_{c}=N_{c}$ without loss of generality, (1) 
can be expressed as

$$
s_{t r}^{k}(t)=\sum_{j=-\infty}^{\infty} s_{j}^{k} b_{\left\lfloor j /\left(N_{f} N_{c}\right)\right\rfloor}^{k} w_{t x}\left(t-j T_{c}\right),
$$

which indicates that IR systems can be regarded as RCDMA systems with generalized spreading sequences, $\left\{s_{j}^{k}\right\}$ [3].

Assume that no data modulation is done during the acquisition stage, that is, $b_{\left\lfloor j /\left(N_{f} N_{c}\right)\right\rfloor}^{1}=1 \forall j^{3}$. In this case, the received signal over a flat fading channel $^{4}$ can be expressed as

$$
r(t)=\sum_{k=1}^{N_{u}} \sum_{j=-\infty}^{\infty} s_{j}^{k} w_{r x}\left(t-j T_{c}-\tau_{k}\right)+\sigma_{n} n(t),
$$

where $\tau_{k}$ is the TOA of user $k\left(\tau_{k}=l T_{c}\right.$, with $l$ being an integer, is assumed for simplicity), $w_{r x}$ is the received UWB pulse and $n(t)$ is white Gaussian noise with unit spectral density.

\section{Sequential Block Search Algorithm}

First, consider the conventional serial search algorithm. In this scheme, the received signal is correlated with a template signal and the output is compared to a threshold. If the output is lower than the threshold, the template signal is shifted by one bin, which corresponds to the resolvable path interval and the correlation with the received signal is obtained again. By this way, the search continues until an output exceeds the threshold.

Each different search location is called a "cell". If the output of the correlation comes from a case where signal path and the template signal are aligned, it is called a signal cell output. Otherwise, it is called a non-signal cell output. The number of cells in the uncertainty region is taken to be $N$. One of these cells is the signal cell while the others are non-signal cells. A false alarm occurs when a non-signal cell output exceeds the threshold. In this case, time $t_{p}$ elapses until the search recovers again. This time is the called penalty time for a false alarm.

For the signal model in (3), the template signal for the serial search can be expressed as follows, assuming no data modulation for the purposes of acquisition:

$$
s_{m_{1}}^{(c)}(t)=\sum_{n=j N_{c}}^{\left(j+m_{1}\right) N_{c}-1} s_{n}^{1} w_{r x}\left(t-n T_{c}\right),
$$

where $m_{1}$ is the number of pulses, over which the correlation is taken.

For the SBS algorithm, there are two different template signals. The first one is similar to the one used in the serial search while the second one is used for searching a block of cells quickly. This latter template signal for the signal model described in (3) can be expressed as follows:

$$
s_{m_{2}}^{(b)}(t)=\sum_{i=0}^{K-1} \sum_{n=j N_{c}}^{\left(j+m_{2}\right) N_{c}-1} s_{n}^{1} w_{r x}\left(t-n T_{c}-i T_{c}\right),
$$

\footnotetext{
${ }^{3}$ Without loss of generality $b_{\left\lfloor j / N_{f} N_{c}\right\rfloor}^{k}=1 \forall j, k$ can be assumed for our purposes.

${ }^{4}$ The results for frequency selective channels can be found in [2].
}

where $K$ is the number of cells in the block and $N=K B$ is assumed with $B$ being the total number of blocks in the uncertainty region. For simplicity, $T_{c}$ is taken as the minimum resolvable path interval.

The output of the correlation between the received signal and the template signal in (6) is used as a quick test to check if the whole block consisting of $K$ cells contains the signal cell or not, while the correlation output of the received signal and the first template in (5) is used in the detailed search of a block.

Let $i$ denote the index of the block that is currently being searched, with $i=1$ initially. Then, the SBS algorithm can be described as follows:

1) Check the $i$ th block using $s_{m_{2}}^{(b)}\left(t-(i-1) K T_{c}\right)$.

2) If the output of the $i$ th block is not higher than the threshold, $\tau_{b}$, then go to step 6 .

3) If the output of the $i$ th block is higher than $\tau_{b}$, then search the block in more detail, i.e. cell-by-cell serial search with threshold $\tau_{s}$ and template $s_{m_{1}}^{(c)}(t)$.

4) If no signal cell is found in the block, go to step 6.

5) If the signal cell is found in the block, DONE.

6) Set $i=(i \bmod B)+1$ and go to step 1 .

Some implicit steps in the algorithm are worth considering. When a false alarm (FA) occurs in the serial search part, the search resumes with the next cell after $C$ time units, which is the penalty time in terms of frame time. So in step 5, "the signal cell is found" means that a correlation output exceeds the threshold without a false alarm. Similarly, in step 4, "no signal cell is found" implies that no correlation output exceeds the threshold without a false alarm.

\section{Statistics of the Decision Variables}

In this section, the statistics of the decision variables will be derived for long correlation intervals.

\section{A. Serial Search Outputs}

In this case, the received signal is correlated with shifted versions of the template signal (5).

$$
y_{i}=\int r(t) s_{m_{1}}^{(c)}\left(t-\Delta_{i}\right) d t
$$

for $i=1, \ldots, N$, where $\Delta_{i}=(i-1) T_{c}$ is the delay of the template signal for the $i$ th cell. The output $y_{i}$ can be expressed as the sum of three terms, $y_{i}=x_{i}+a_{i}+n_{i}$, where

$$
\begin{array}{ll}
x_{i}: \text { Signal part/Self interference, } \\
a_{i}: \text { Multiple access interference (MAI), } \\
n_{i}: \text { Output noise. }
\end{array}
$$

The output noise can be shown to be distributed as $\mathcal{N}\left(0, m_{1} \sigma_{n}^{2}\right)$. The first term $x_{i}$ can be expressed as follows depending on the signal/non-signal cell situation:

1) Signal Cell Case: In this case, the template signal (5) and the received signal are aligned. Therefore, the correlation over $m_{1}$ pulses gives the following signal part

$$
x_{l_{s}}=\sum_{j=1}^{m_{1} N_{c}} s_{j}^{2}=m_{1}
$$


since $s_{j}$ is non-zero $( \pm 1)$ only once in $N_{c}$ consecutive cells. Note that $l_{s}$ is the index of the signal cell.

2) Non-Signal Cell Case: Again consider a correlation over $m_{1}$ pulses. In this case, the received signal and the template signal in (5) are misaligned by one or more cells. Then, the self interference part of the output of the correlation can be expressed as

$x_{i}=\sum_{n=j N_{c}}^{\left(j+m_{1}\right) N_{c}-1} \int s_{n}^{1} w_{r x}\left(t-n T_{c}-\Delta_{i}\right) \sum_{m=-\infty}^{\infty} s_{m}^{1} w_{r x}\left(t-m T_{c}-\tau_{1}\right) d t$

for $i=1, \ldots, l_{s}-1, l_{s}+1, \ldots, N$.

Note that since the outer summation is over $m_{1} N_{c}$ terms, $s_{n}^{1}$ will be non-zero $( \pm 1)$ just $m_{1}$ times. So (9) can be considered as the sum of $m_{1}$ separate correlations, each of which denotes the output due to one pulse of the template signal. If we denote each of these terms by a random variable $x_{i l}$ for $l=1, \ldots, m_{1}$, the self interference part of the output can be expressed as

$$
x_{i}=\sum_{l=1}^{m_{1}} x_{i l}
$$

where

$x_{i l}=\int d_{j+l-1}^{1} w_{r x}\left(t-n T_{c}-\Delta_{i}\right) \sum_{m=-\infty}^{\infty} s_{m}^{1} w_{r x}\left(t-m T_{c}-\tau_{1}\right) d t$.

Now the aim is to find the probability distribution of $x_{i l}$ for each $l$. To this end, assume that the amount of misalignment between the received signal and the template signal is an integer multiple of $T_{c}$ for all non-signal cell situations, that is, $\tau_{1}-\Delta_{i}$ is an integer multiple of $T_{c}$. Let $\gamma_{i}=\left|\tau_{1}-\Delta_{i}\right| / T_{c}$ be the integer representing the misalignment between the delay of the $i$ th template signal and the received signal in terms of $T_{c}$, which can also be interpreted as the distance between the $i$ th non-signal cell and the signal cell.

Note that since each $x_{i l}$ is the output of the correlation between one pulse in the template signal and the received signal, it is obvious that $x_{i 1}, \ldots, x_{i m_{1}}$ are identically distributed.

First, consider the case where $\gamma_{i}<N_{c}$, that is, the misalignment between the received and the template signals is less than $N_{c} T_{c}$ seconds. In this case, $x_{i l}$ is non-zero only when the $l$ th pulse of the template signal overlaps with any of the received signal pulses. Since $\gamma_{i}<N_{c}$, two pulses can overlap only if they are in frames which are next to each other. Therefore, for the $l$ th pulse of the template signal to overlap with a pulse of the received signal, the pulse should be in one of the $\gamma_{i}$ positions close to the next frame and the next pulse in that frame should be in the position, to which this $l$ th pulse is shifted. The probability that these two events occur simultaneously is $\gamma_{i} / N_{c}^{2}$. Therefore, with probability $\gamma_{i} / N_{c}^{2}$, the output is non-zero. This non-zero output is the multiplication of two consecutive coding values: $d_{j+l-1}^{1}$ and $d_{j+l}^{1}$ or $d_{j+l-1}^{1}$ and $d_{j+l-2}^{1}$, where $d_{j}^{k}$ is as in (1) and related to the spreading sequence as shown in (2). They take values \pm 1 with equal probability and are independent of each other by definition. Therefore, the probability mass function for $x_{i l}$ for $l=1,,, . m_{1}$ can be expressed as

$$
p_{x_{i l}}(x)= \begin{cases}\gamma_{i} / 2 N_{c}^{2} & x= \pm 1 \\ 1-\gamma_{i} / N_{c}^{2} & x=0\end{cases}
$$

From (12), it is seen that $\mathrm{E}\left\{x_{i l}\right\}=0$ and $\operatorname{Var}\left\{x_{i l}\right\}=\gamma_{i} / N_{c}^{2}$ for $l=1, \ldots, m_{1}$.

Although the stationary sequence $\left\{x_{i l}\right\}_{l=1}^{m_{1}}$ is not independent, it is a 1-dependent random sequence ${ }^{4}$ and hence a $t \phi$-mixing random sequence satisfying the conditions in [4]. Therefore, central limit theorem (CLT) can be applied and for sufficiently large $m_{1}$ the probability distribution of $x_{i}$ can be approximately expressed as $\mathcal{N}\left(0, m_{1} \sigma^{2}\right)$ where

$$
\sigma^{2}=\mathrm{E}\left\{x_{i 1}^{2}\right\}+2 \sum_{j=1}^{\infty} \mathrm{E}\left\{x_{i 1} x_{i(1+j)}\right\} .
$$

It is easy to show that the cross-correlation term is zero. Therefore, the following asymptotic distribution is obtained:

$$
x_{i} \sim \mathcal{N}\left(0, m_{1} \gamma_{i} / N_{c}^{2}\right) \quad \gamma_{i}<N_{c} .
$$

When $\gamma_{i} \geq N_{c}$, we can follow similar approaches and show that

$$
x_{i} \sim \mathcal{N}\left(0, m_{1} / N_{c}\right) \quad \gamma_{i} \geq N_{c} .
$$

For the MAI term, we can similarly show that for large correlation intervals, it can be approximated by the following Gaussian random variable:

$$
a_{i} \sim \mathcal{N}\left(0,\left(N_{u}-1\right) m_{1} / N_{c}\right)
$$

\section{B. Block Search Outputs}

In this case, the received signal is correlated with shifted versions of the template signal (6). The block containing the signal cell is called the signal block while the blocks consisting of all non-signal cells are called non-signal blocks.

The correlation output can be expressed as

$$
y_{i}=\int r(t) s_{m_{2}}^{(b)}\left(t-\Delta_{i}^{\prime}\right) d t,
$$

for $i=1, \ldots, B$ with $\Delta_{i}^{\prime}=(i-1) K T_{c}$ and $N=K B$.

Again the output can be expressed as a sum of three terms, $y_{i}=x_{i}+a_{i}+n_{i}$, where $x_{i}$ is the signal part or self interference term, $a_{i}$ is MAI term and $n_{i}$ is the output noise. Note that $n_{i} \sim \mathcal{N}\left(0, m_{2} K \sigma_{n}^{2}\right)$. Also by similar CLT arguments, $a_{i} \sim$ $\mathcal{N}\left(0,\left(N_{u}-1\right) m_{2} K / N_{c}\right)$.

For $x_{i}$, we have the following results:

1) Non-Signal Block Case: In this case, all of the $K$ cells in the block are non-signal cells. The following lemma states that under some situations, the probability density function of the self interference terms of the non-signal block output can be approximated by a Gaussian density function.

Lemma 4.1: Assuming that each of the $K$ cells in the block is away from the signal cell by more than $N_{c}$ cells, the probability distribution of the self interference term of the non-signal block output can be approximated by the following Gaussian distribution for large values of correlation interval

\footnotetext{
${ }^{4}\left(X_{1}, \ldots, X_{i}\right)$ and $\left(X_{i+j}, \ldots\right)$ are independent whenever $j>1$.
} 
$m_{2}$

$$
x_{i} \sim \mathcal{N}\left(0, m_{2} K / N_{c}\right),
$$

for $i=1, \ldots, l_{b}-1, l_{b}+1, \ldots, B$, where $l_{b}$ is the index of the signal block.

Proof See [2].

2) Signal Block Case: In this case, the signal cell is in the block and the remaining $K-1$ cells are non-signal cells. For correlation over $m_{2}$ pulses, the distribution of the signal part of the signal block output can again be approximated by a Gaussian distribution under some conditions. We state the result for the simple case where $2 \leq K<N_{c}$ (see [2] for the general expression).

Lemma 4.2: For $2 \leq K<N_{c}$, the probability distribution of the signal part of the signal block output can be approximated by the following Gaussian distribution for large values of the correlation interval $m_{2}$

$$
x_{l_{b}} \sim \mathcal{N}\left(m_{2}, m_{2}\left[\frac{K^{2}-1}{3 N_{c}^{2}}+\frac{n(n+1)(3 K-4 n-2)}{3 K N_{c}^{2}}\right]\right),
$$

where $n=\left\lfloor\frac{K-1}{2}\right\rfloor$. Or, in a more reduced form, $x_{l_{b}} \sim$ $\mathcal{N}\left(m_{2}, m_{2}\left(5 K^{2}-c\right) /\left(12 N_{c}^{2}\right)\right)$ where $c=5$ if $K$ is odd and $c=8$ for even $K$.

Proof See [2].

\section{ACQuisition Time AnAlysis}

Using the approximate probability distributions derived in the previous section, the probabilities of false alarms and miss detections in different scenarios can be expressed using Qfunctions. Then, the aim becomes the derivation of the mean acquisition time (MAT) formulas.

It is shown in [1] that when the signal cell location is uniformly distributed through the uncertainty region, the MAT for the serial search in a flat fading channel can be expressed approximately as

$$
\mathrm{E}\left\{T_{\text {serial }}\right\} \approx \frac{N\left(2-P_{D}\right)\left(1+C P_{F A} / m_{1}\right)}{2 P_{D}} \cdot m_{1}
$$

for large $N$ values, where $N$ is the number of cells in the uncertainty region, $P_{D}$ is the probability of detection of the signal path and $P_{F A}$ is the probability of false alarm. The correlation over one frame is taken to be one unit time. When a false alarm occurs, $C$ penalty time units elapse before the search continues with the next cell.

Using the signal flow graph approach in [1], we can show that for large number of blocks $B$, the MAT for the SBS algorithm can be expressed as

$$
\mathrm{E}\left\{T_{S B S}\right\} \approx \frac{\left[m_{2}+K P_{N}\left(m_{1}+C P_{F A}\right)\right]\left(2-P_{S} P_{D}\right) B}{2 P_{S} P_{D}},
$$

where $P_{N}$ is the probability that a non-signal block output exceeds the threshold and $P_{S}$ is the probability that a signal block output exceeds the threshold.

Define asymptotic gain as the ratio of the serial search MAT to the SBS MAT under the limiting conditions $\sigma_{n}^{2} \longrightarrow 0$ and $N_{c} \longrightarrow \infty$. It can be shown [2] that

$$
G=10 \log \frac{N / 2}{\left.(B / 2+K / 2)\right|_{B=K=\sqrt{N}}}=10 \log \frac{\sqrt{N}}{2} .
$$

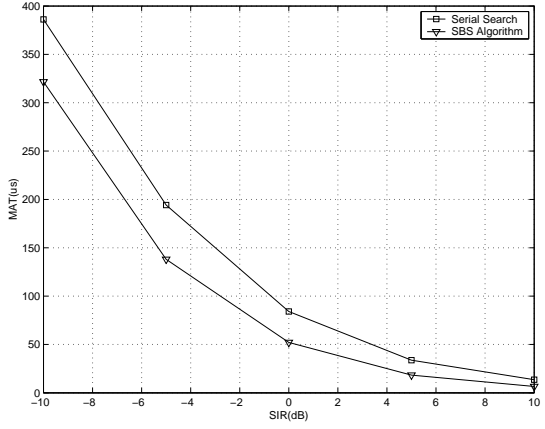

Fig. 1. Mean acquisition times for different algorithms.

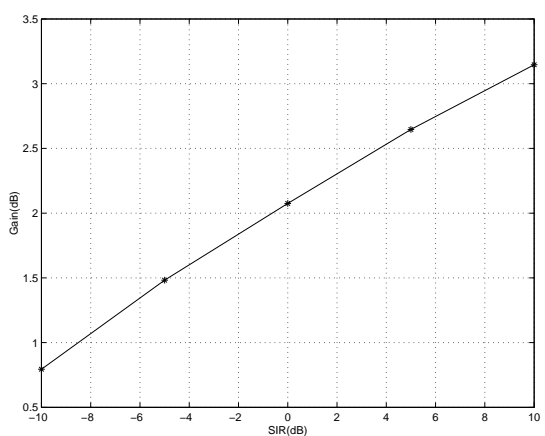

Fig. 2. Gain for different SIR values.

In other words, in the ideal conditions, the gain increases as the number of cells in the uncertainty region increases.

For realistic situations, we perform some simulation experiments to compare the algorithms. Figure 1 compares the MATs of serial search and SBS algorithms for different $\operatorname{SIR}^{5}$ values when the number of cells in the uncertainty region, $N$, is 360 , chip interval $T_{c}$ is $0.25 \mathrm{~ns}$, false alarm penalty is 100 unit time and there are 10 users in the system. Here, we can see that SBS algorithm has lower mean acquisition times and the gain increases as SIR increases as shown in Figure 2.

\section{CONCLUSION}

In this paper, a rapid acquisition technique has been proposed and its performance has been evaluated. It comparison to conventional serial search technique shows the efficiency of the algorithm.

\section{REFERENCES}

[1] A. Polydoros and C. L. Weber, "A unified approach to serial search spread-spectrum code acquistion-Part I: General Theory," IEEE Trans. Comm., vol. COM-32, pp. 542-549, May 1984.

[2] S. Gezici, E. Fishler, H. Kobayashi, H. V. Poor, and A. F. Molisch, "Rapid Acquisition for Impulse Radio", in preparation.

[3] E. Fishler and H. V. Poor, "On the tradeoff between two types of processing gain," 40th Annual Allerton Conference on Communication, Control, and Computing, 2002.

[4] P. Bilingsly, "Probability and Measure," John Wiley \& Sons, New York, 2nd edition, 1986.

${ }^{5}$ For unit pulse energy, SIR $=-10 \log \left(\sigma_{n}^{2}+\left(N_{u}-1\right) / N_{c}\right)$ is used as the definition of the SIR. 